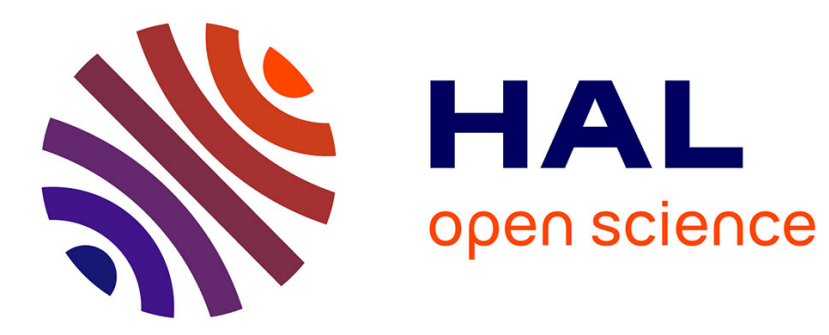

\title{
Convectively driven dynamo action in the quiet Sun
}

Paul Bushby, Benjamin Favier, Michael R. E. Proctor, Nigel Weiss

\section{To cite this version:}

Paul Bushby, Benjamin Favier, Michael R. E. Proctor, Nigel Weiss. Convectively driven dynamo action in the quiet Sun. Geophysical and Astrophysical Fluid Dynamics, 2012, 106 (4-5), pp.508-523. 10.1080/03091929.2011.638067 . hal-01287831

\section{HAL Id: hal-01287831 \\ https://hal.science/hal-01287831}

Submitted on 25 Oct 2018

HAL is a multi-disciplinary open access archive for the deposit and dissemination of scientific research documents, whether they are published or not. The documents may come from teaching and research institutions in France or abroad, or from public or private research centers.
L'archive ouverte pluridisciplinaire HAL, est destinée au dépôt et à la diffusion de documents scientifiques de niveau recherche, publiés ou non, émanant des établissements d'enseignement et de recherche français ou étrangers, des laboratoires publics ou privés. 


\title{
Convectively-driven dynamo action in the quiet Sun
}

\author{
P. J. Bushby†*, B. Favier†, M. R. E. Proctorł and N. O. Weiss $\ddagger$ \\ †School of Mathematics and Statistics, Newcastle University, Newcastle Upon Tyne, NE1 7RU, U.K. \\ $\ddagger$ DAMTP, Centre for Mathematical Sciences, Wilberforce Road, Cambridge, CB3 0WA, U.K.
}

(May 2011)

\begin{abstract}
Observations of the quiet solar surface indicate that localised concentrations of vertical magnetic flux tend to accumulate in the convective downflows. Furthermore, there is some evidence to suggest that mesogranular boundaries are preferred locations for the formation of these flux concentrations. This implies that these magnetic fields are organised on scales that are larger than the granular scale. One possible explanation for the existence of quiet Sun magnetic features is that they are continuously regenerated by the near-surface convective motions. Motivated by this, we consider dynamo action in a local Cartesian model of convection in a compressible electrically-conducting fluid. The horizontal scale of this domain is large enough to model mesoscale behaviour. Dynamo action occurs provided that the magnetic Reynolds number exceeds some critical value. In the kinematic regime the presence of mesogranules seems to be beneficial for dynamo action: compared to similar smaller aspect ratio calculations, we find higher kinematic growth rates for the magnetic energy, as well as a lower value for the critical magnetic Reynolds number. In the nonlinear regime the peak magnetic field strengths compare very favourably to observations, greatly exceeding the equipartition value at the surface, as observed in the quiet Sun. However, there is no evidence to suggest that the presence of mesogranules significantly increases the saturation level of the dynamo in this nonlinear regime, which (in the highest magnetic Reynolds number case) is comparable to that found in similar calculations in smaller domains.
\end{abstract}

Keywords: Dynamo; Convection; Magnetic fields

\section{Introduction}

In the quiet Sun, near-surface convective motions form a characteristic granular pattern. The central bright region of each granule corresponds to a convective upflow, whereas the dark intergranular lanes correspond to convective downflows. A typical granule has a diameter of approximately $1 \mathrm{Mm}$ and a lifetime of a few minutes (see, for example, Stix 2004). Although regions of quiet Sun are characterised by an absence of large magnetic features, small-scale concentrations of positive and negative vertical magnetic flux do accumulate in the intergranular lanes (Lin \& Rimmele 1999). This tendency for magnetic flux to be associated with the convective downflows is particularly apparent in G-band images of the solar surface (see, for example, Sánchez Almeida et al. 2010), where strong magnetic fields (of either polarity) show up as bright points in the intensity map. Typical field strengths in these bright points can often exceed a Kilogauss, which implies that the local magnetic energy density of these intense flux concentrations significantly exceeds the mean kinetic energy density of the surrounding convective motions (Galloway et al. 1977). The distribution of magnetic fields in the quiet Sun has been the subject of numerous recent studies (e.g. Domínguez Cerdeña et al. 2006, Parnell et al. 2009; see also de Wijn et al. 2009, and references therein).

Certain features of quiet Sun magnetic fields are now well understood. The tendency for convective eddies to expel magnetic fields (Weiss 1966) explains why intergranular lanes are preferred locations for concentrations of vertical magnetic flux. The observed Kilogauss-strength magnetic fields can also be accounted for if the gas density within these intense flux concentrations is small. It has been shown in idealised models of thin magnetic flux tubes (see, for example, Spruit 1979), as well as in more realistic models of magnetoconvection (see, for example, Bushby et al. 2008), that convective motions naturally lead

*Corresponding author. Email: paul.bushby@ncl.ac.uk 
to the partial evacuation of regions of vertical magnetic flux. Furthermore, this process has recently been observed directly at the solar surface (Nagata et al. 2008). If the gas density within the partially-evacuated magnetic region is sufficiently small, the magnetic pressure that is associated with a Kilogauss-strength magnetic field can be balanced by the gas pressure of the surrounding non-magnetic fluid. Hence, provided that there is a continuous supply of magnetic flux at the solar surface, the presence of superequipartition magnetic fields in the quiet Sun intergranular lanes is relatively straightforward to explain. The crucial question, however, is where does this flux actually come from? One possibility is that quiet Sun magnetic fields are simply reprocessed fragments of magnetic flux that have risen to the surface from deep within the solar interior. Indeed, models of magnetoconvection, which study the interaction of convective flows with an imposed magnetic field, have successfully reproduced a number of features of quiet Sun magnetic fields (see, for example, Khomenko et al. 2005, Stein \& Nordlund 2006, Bushby et al. 2008). The other plausible explanation for the origin of quiet Sun magnetic fields is that some of the observed fields are generated locally by the near-surface convective motions.

Convectively-driven dynamos have been studied extensively in recent years. Cartesian models of turbulent Boussinesq convection, in an electrically-conducting fluid, have demonstrated that dynamo action is possible in such systems provided that the inductive effects of the fluid motions outweigh the dissipative effects of magnetic diffusion (Meneguzzi \& Pouquet 1989, Cattaneo 1999). The magnetic fields that are produced in models of this type are small-scale and intermittent. Furthermore, with appropriate boundary conditions, the vertical component of the magnetic field at the surface forms mixed polarity flux concentrations at the convective downflows. Hence, results from these simulations can be related (in a qualitative way) to solar observations. Clearly, one of the main limitations of the Boussinesq approximation is that the effects of compressibility are neglected. Compressibility does play an important role in the near-surface convective motions and, as already discussed, it is difficult to explain the observed magnetic field strengths unless there is a significant reduction in the fluid density within the strongest magnetic flux concentrations. Compressible convection is more challenging numerically than its Boussinesq counterpart. Nevertheless, a number of recent numerical studies have demonstrated that it is possible to excite a convectively-driven dynamo in a compressible layer of fluid (Abbett 2007, Vögler \& Schüssler 2007, Käpylä et al. 2008, Schüssler \& Vögler 2008, Brummell et al. 2010, Bushby et al. 2010, Pietarila Graham et al. 2010, Bushby et al. 2011). As in the Boussinesq case, all these calculations produce magnetic field distributions that are similar to those observed in the quiet Sun.

One of the main limitations of local Cartesian models is that only a small region of the solar surface can be simulated. Indeed, in most of the compressible models that are described above the horizontal extent of the computational domain is only a factor of two or three times larger than the horizontal scale of the simulated granules. This is a significant point because observations of the quiet Sun reveal organised convective motions on scales that are considerably larger than the granular scale. For example, supergranules have a typical length scale of approximately $30 \mathrm{Mm}$ and a typical lifetime of approximately one day (see, for example, Ricutord \& Rincon 2010). Unfortunately, it is not yet feasible to carry out simulations that can properly describe the evolution of convection over a supergranular scale. On a slightly smaller scale there are also mesogranular convective cells (see, for example, November et al. 1981, Muller et al. 1992, Shine et al. 2000, Domínguez Cerdeña 2003), with typical spatial scales of between 5 and $10 \mathrm{Mm}$ and typical lifetimes of approximately two hours. Mesogranules do appear to play a significant role in the evolution of quiet Sun magnetic fields, with flux concentrations accumulating preferentially in the intergranular lanes that coincide with boundaries of mesogranular cells (Yelles Chaouche et al. 2011). The explanation for the origin of mesogranular motions is still under debate (see, for example, Cattaneo et al. 2001, Rincon et al. 2005). What is clear, however, is that it is possible to simulate mesogranular convection in large aspect ratio domains (Rincon et al. 2005). Simulations of dynamo action in large aspect ratio Boussinesq convection (Cattaneo et al. 2001) do support the idea that concentrations of vertical magnetic flux tend to be associated with the mesogranular boundaries. However, this has not yet been studied in fully compressible convection. That is the aim of the present paper. 


\section{$2 \quad$ Model}

\section{$2.1 \quad$ Governing equations}

We consider convection in a plane layer of electrically-conducting, compressible fluid. This fluid occupies a Cartesian domain of large aspect ratio, defined by $0 \leq x \leq 10 d, 0 \leq y \leq 10 d$ and $0 \leq z \leq d$, with the constant gravitational acceleration (denoted by $g$ ) acting in the positive $z$-direction. The upper boundary of the domain (i.e. $z=0$ ) is held at fixed temperature, $T=T_{0}$, whilst we take a fixed value of $T=T_{0}+\Delta T$ at $z=d$. A positive value of $\Delta T$ implies that the layer is heated from below. The upper and lower boundaries are also assumed to be impermeable and stress-free. Any magnetic fields that are present are also constrained to be vertical at $z=0$ and $z=d$. Periodic boundary conditions are imposed in each of the horizontal directions. This Cartesian model is intended to be an idealised representation of a localised region of convection in the quiet Sun.

For simplicity, we assume that the fluid has constant values for the thermal conductivity $K$, the shear viscosity $\mu$, the magnetic diffusivity $\eta$, the magnetic permeability $\mu_{0}$ and the specific heat capacities, $c_{P}$ and $c_{V}$. Before writing down the governing equations, we first make this system dimensionless (adopting scalings that are similar to those described by Matthews et al. 1995). All length-scales are expressed in terms of the layer depth $d$. The temperature is scaled in terms of $T_{0}$, whilst the density $\rho$ is scaled by $\rho_{0}$ (the density at the upper surface in the absence of convective motions). Defining $R_{*}=c_{P}-c_{V}$ to be the gas constant, we scale the velocity $\boldsymbol{u}$ by the isothermal sound speed at the top of the layer, $\left(R_{*} T_{0}\right)^{1 / 2}$. It is therefore natural to scale time by $d /\left(R_{*} T_{0}\right)^{1 / 2}$. Finally, we scale the magnetic field $\boldsymbol{B}$ by $\left(\mu_{0} \rho_{0} R_{*} T_{0}\right)^{1 / 2}$. This scaling implies that the Alfvén speed at the top of the layer is also expressed in terms of the isothermal sound speed.

Having non-dimensionalised this system, the governing equations can be written in the following form:

$$
\begin{aligned}
\frac{\partial \rho}{\partial t}+\boldsymbol{u} \cdot \boldsymbol{\nabla} \rho & =-\rho \boldsymbol{\nabla} \cdot \boldsymbol{u}, \\
\rho\left(\frac{\partial \boldsymbol{u}}{\partial t}+\boldsymbol{u} \cdot \boldsymbol{\nabla} \boldsymbol{u}\right) & =-\boldsymbol{\nabla} P+(\boldsymbol{\nabla} \times \boldsymbol{B}) \times \boldsymbol{B}+\theta(m+1) \rho \hat{\boldsymbol{z}}+\kappa \sigma \nabla \cdot \boldsymbol{\tau}, \\
\frac{\partial T}{\partial t}+\boldsymbol{u} \cdot \boldsymbol{\nabla} T & =-(\gamma-1) T \boldsymbol{\nabla} \cdot \boldsymbol{u}+\frac{\kappa \gamma}{\rho} \nabla^{2} T+\frac{\kappa(\gamma-1)}{\rho}\left(\sigma \tau^{2} / 2+\zeta_{0}|\boldsymbol{\nabla} \times \boldsymbol{B}|^{2}\right), \\
\frac{\partial \boldsymbol{B}}{\partial t} & =\boldsymbol{\nabla} \times\left(\boldsymbol{u} \times \boldsymbol{B}-\kappa \zeta_{0} \boldsymbol{\nabla} \times \boldsymbol{B}\right),
\end{aligned}
$$

where $\boldsymbol{B}$ satisfies $\boldsymbol{\nabla} \cdot \boldsymbol{B}=0$. In this illustrative model, we assume that the pressure, $P$, satisfies an appropriate equation of state for a perfect gas, $P=\rho T$, whilst the components of the tensor, $\boldsymbol{\tau}$, are given by

$$
\tau_{i j}=\frac{\partial u_{i}}{\partial x_{j}}+\frac{\partial u_{j}}{\partial x_{i}}-\frac{2}{3} \frac{\partial u_{k}}{\partial x_{k}} \delta_{i j}
$$

These governing equations are solved numerically, using a modified version of the code that was described by Matthews et al. (1995). All horizontal derivatives are evaluated in Fourier space. Vertical derivatives are estimated using a fourth-order finite difference scheme, adopting an upwind stencil for the advective terms within the code. A poloidal-toroidal decomposition is used for the magnetic field so as to ensure that the field remains solenoidal. Time-stepping is carried out using an explicit third-order Adams-Bashforth scheme, with a variable time-step. The code is parallelised using MPI. Unless otherwise stated, all calculations are based upon a computational mesh containing $512 \times 512 \times 96$ grid points. 
Table 1. Non-dimensional parameters for the dynamo model

\begin{tabular}{cccc}
\hline Parameter & Description & Definition & Values \\
\hline$\gamma$ & Ratio of specific heat capacities & $c_{P} / c_{V}$ & $5 / 3$ \\
$m$ & Polytropic index & $\left(g d / R_{*} \Delta T\right)-1$ & 1.0 \\
$\theta$ & Thermal stratification & $\Delta T / T_{0}$ & 3.0 \\
$\sigma$ & Prandtl number & $\mu c_{P} / K$ & 1.0 \\
$\kappa$ & Dimensionless thermal diffusivity & $K / \rho_{0} d c_{P}\left(R_{*} T_{0}\right)^{1 / 2}$ & 0.00548 \\
$\zeta_{0}$ & Ratio of magnetic to thermal diffusivities & $\eta \rho_{0} c_{P} / K$ & $0.15,0.35,0.55$ \\
\hline
\end{tabular}

\subsection{Parameter values and initial conditions}

The various non-dimensional parameters that appear in this model are defined in Table 1. A choice of $\gamma=5 / 3$ is appropriate for a monatomic gas, whilst a polytropic index of $m=1.0$ implies that the layer is convectively unstable. A thermal stratification of $\theta=3$ implies that the temperature varies by a factor of 4 across the layer. The effects of varying $\theta$ are discussed in Bushby et al. (2010). A Prandtl number of $\sigma=1.0$ is not particularly appropriate for the solar photosphere (where $\sigma \ll 1$ ), but a realistic value is computationally inaccessible, so this is a pragmatic choice that has been made for numerical convenience. For the dimensionless thermal diffusivity, we choose a value of $\kappa=0.00548$. This implies that the mid-layer Rayleigh number (see, for example, Matthews et al. 1995) is approximately $3 \times 10^{5}$. In the absence of a magnetic field this is more than two orders of magnitude larger than the critical Rayleigh number for the onset of convection in an initially hydrostatic polytropic layer. The resulting motions, which are illustrated in Figure 1, are vigorous and time-dependent. This statistically-steady hydrodynamic flow is used as an initial condition for all the dynamo calculations that are described in this paper. At some instant in time, a seed magnetic field (with zero net vertical flux) is introduced into the flow. At the same time, a value of $\zeta_{0}$ is selected (see Table 1). With all other parameters fixed, the value of $\zeta_{0}$ determines the magnitude of the magnetic diffusivity. Dynamo action is only expected in the low $\zeta_{0}$ regime.

In calculations of this type, it is useful to consider the Reynolds number of the flow. There are various possible definitions for the Reynolds number, although it is natural to use the depth of the layer (which equals unity in these non-dimensional units) and the rms velocity $\left(U_{r m s}\right)$ as characteristic length and velocity scales. Because the kinematic viscosity is inversely proportional to the density, the Reynolds number is actually a function of depth. We choose to quote a mid-layer value, $\mathcal{R} e=\rho_{\text {mid }} U_{r m s} / \kappa \sigma$ (where $\rho_{\text {mid }}$ is the mean density at $z=0.5$ ). For the parameter values that are given in Table 1, the resulting flow has a Reynolds number of approximately $\mathcal{R} e=155$. We can make an analogous definition for the magnetic Reynolds number, $\mathcal{R} m=U_{r m s} / \kappa \zeta_{0}$. For the three values of $\zeta_{0}$ that are given in Table $1, \mathcal{R} m=430,185$ and 115. Another useful parameter to consider is the convective turnover time, $\tau_{c}$. Defining this quantity to be the depth of the box divided by the rms velocity (see, for example, Cattaneo 1999), we obtain a value of $\tau_{c} \approx 3$ (dimensionless) time units. One further parameter that is of interest in this system is the magnetic Prandtl number, $P m=\mathcal{R} m / \mathcal{R} e$. In the solar photosphere, $P m \ll 1$, whereas it is of order unity in these calculations (due to computational constraints). This issue is discussed in more detail in the conclusions.

\section{Numerical results}

\subsection{Hydrodynamic convection}

Before investigating the dynamo properties of this system, we first analyse the hydrodynamic behaviour of the flow that is illustrated in Figure 1. Near the upper surface, it is apparent that the convective motions form a granular pattern, with broad, warm upflows, surrounded by a network of cooler downflows. Clearly this is reminiscent of the observed flows at the solar surface. At the mid-layer of the computational domain, as shown in Figure 1(c), it is more difficult to discern a pattern of motion that could be related directly to the surface granulation. However, those convective downflows that are strong enough to persist down to this depth form a network of cooler lanes that is suggestive of a mesogranular scale. Certainly, the dominant convective downflows at this depth appear to enclose larger regions than the intergranular downflows near the upper surface of the domain. Near the lower boundary of the computational domain, the flow is highly 


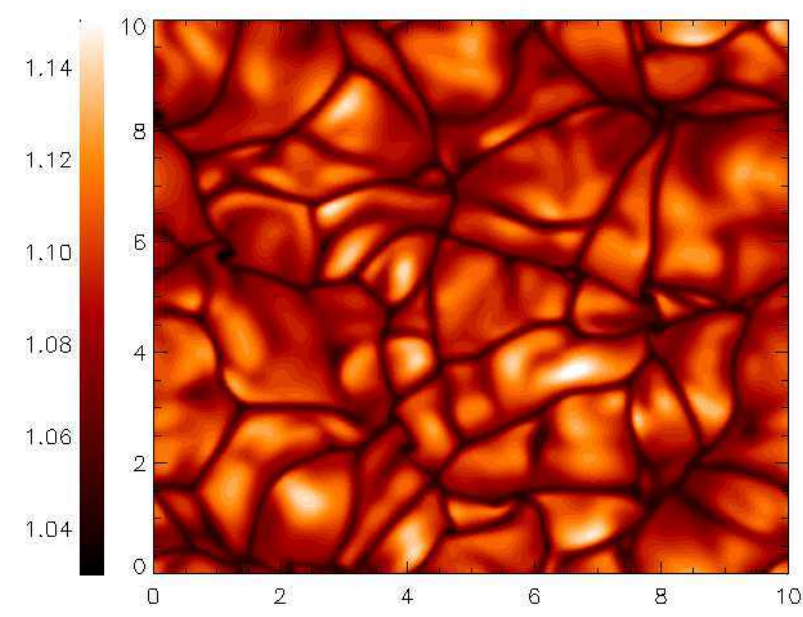

(a) $z=0.02$

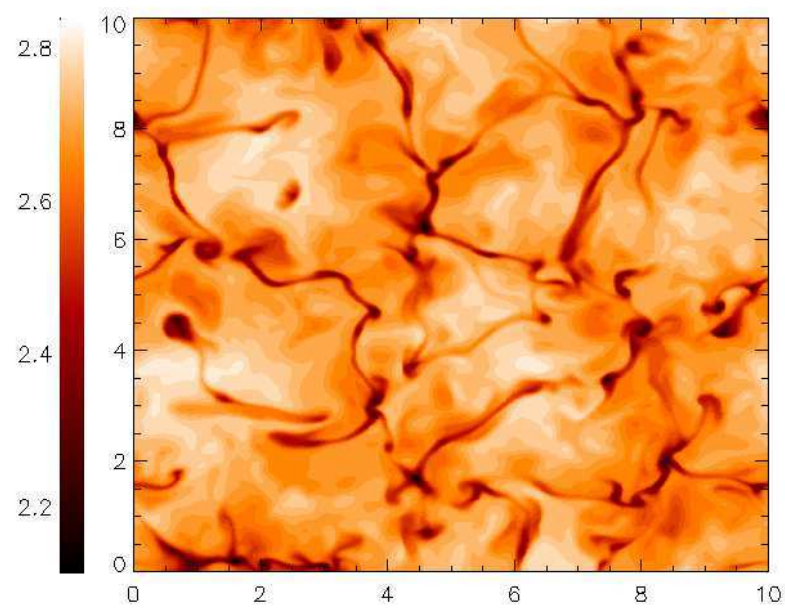

(c) $z=0.5$

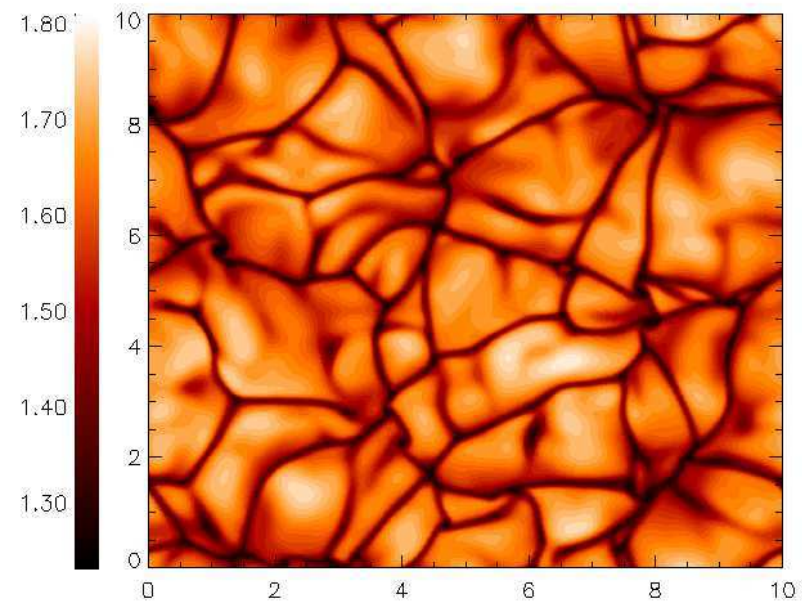

(b) $z=0.1$

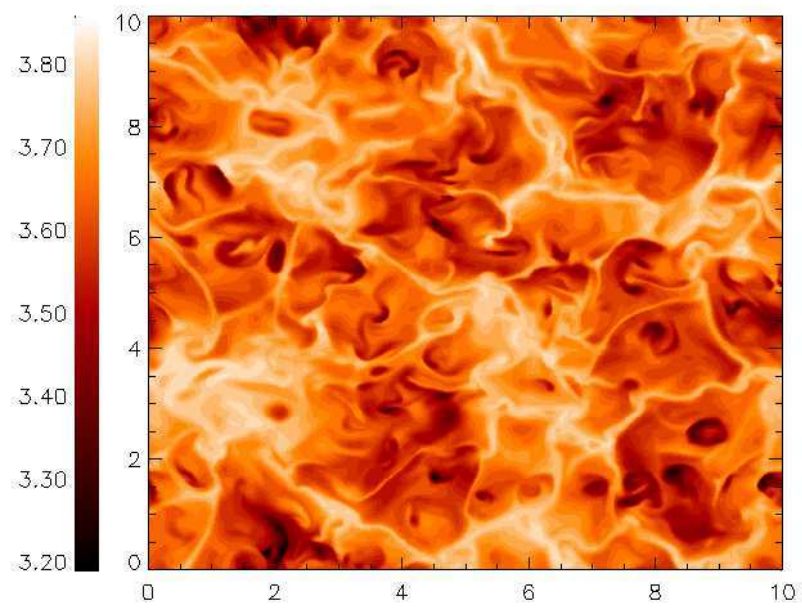

(d) $z=0.9$

Figure 1. (colour online) Statistically-steady hydrodynamic convection at $t=232$ (just before the magnetic field is introduced). These plots show the temperature distribution in four different horizontal planes. Bright (dark) regions correspond to high (low) temperatures.

disordered, resulting in a complex temperature distribution that fluctuates on small scales. Note however that some persistent features can be observed at this depth as the simulation is evolved.

Although a visual analysis of the depth-dependence of the temperature distribution does give some indication of mesoscale behaviour in this system, this is not the only means we have of diagnosing the presence of mesogranules. In their simulation of hydrodynamic convection, Rincon et al. (2005) found that the peak in the momentum spectrum corresponded to the mesogranular scale. Therefore, we can use spectra to determine whether or not mesogranules are present. For each value of $z$, it is possible to define the kinetic energy spectrum, $E_{K}(k, z)$, based on the magnitude of the horizontal wavenumber, $k$. We compute this quantity as follows:

$$
E_{K}(k, z)=\frac{1}{4} \sum_{k_{x}} \sum_{k_{y}}\left[\widehat{\boldsymbol{u}}\left(k_{x}, k_{y}, z\right) \cdot \widehat{\rho \boldsymbol{u}}^{*}\left(k_{x}, k_{y}, z\right)+\widehat{\boldsymbol{u}}^{*}\left(k_{x}, k_{y}, z\right) \cdot \widehat{\rho \boldsymbol{u}}\left(k_{x}, k_{y}, z\right)\right]
$$

where $\hat{\boldsymbol{u}}\left(k_{x}, k_{y}, z\right)$ is the two-dimensional Fourier transform of $\boldsymbol{u}(x, y, z)$, with the star denoting the corresponding complex conjugate, whilst the summation is over all values of $k_{x}$ and $k_{y}$ such that $k^{2}=k_{x}^{2}+k_{y}^{2}$. 

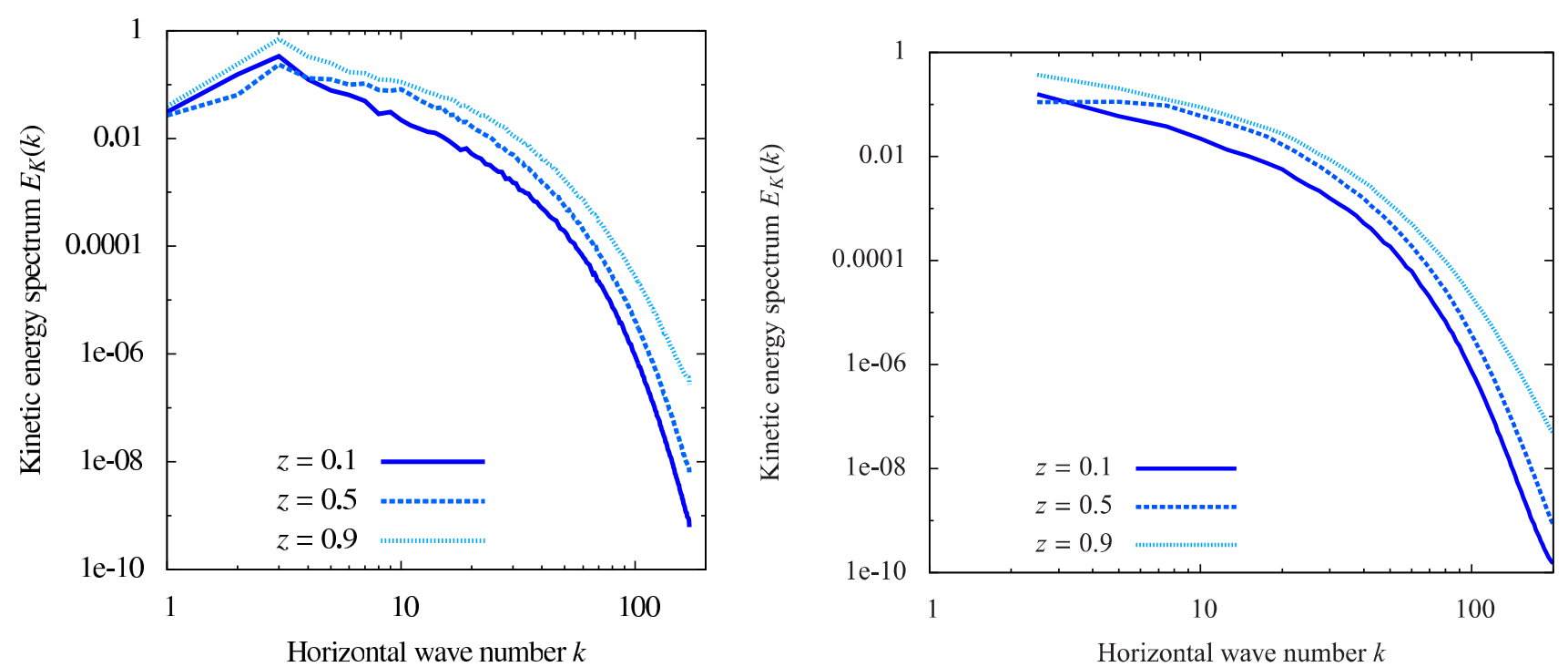

Figure 2. The kinetic energy spectrum for hydrodynamic convection for $z=0.1$ (solid line), $z=0.5$ (long dashed line) and $z=0.9$ (short dashed line). Left: The spectrum for the $10 \times 10 \times 1$ computational domain that is considered in this paper. Right: The corresponding energy spectrum for a comparable $4 \times 4 \times 1$ simulation. Note that the wavenumbers are defined in the same way in both plots (with $k=1$ corresponding to the lowest wavenumber mode in the $10 \times 10 \times 1$ simulation).

On the left-hand side of Figure 2, we show the time-average of the kinetic energy spectrum at depths $z=0.1, z=0.5$ and $z=0.9$. For the purpose of comparison, the right-hand side of Figure 2 shows the corresponding kinetic energy spectrum for a calculation that was carried out with the same parameters, but this time in a smaller $4 \times 4 \times 1$ computational domain (see, for example, Bushby et al. 2011). The Reynolds numbers (and rms velocities) are comparable in the two cases. Note that the numerical resolution in the smaller aspect ratio calculation was $256 \times 256 \times 96$ mesh points. As is evident from Figure 2, the kinetic energy spectrum in the $4 \times 4 \times 1$ calculation tends to decrease monotonically with increasing wavenumber at $z=0.1$ and $z=0.9$. Slightly different behaviour is seen at the mid-layer of the domain (i.e. at $z=0.5$ ), where the spectrum is rather flat at low wavenumbers. However, even in this case, there is no clear peak in the spectrum that would correspond to a well-defined granular scale. Individual granules tend to grow as they evolve in time (until they are eventually swallowed up by the motions of the neighbouring granules), so it is not surprising that a wide range of convective scales is observed. Although there is also no indication of a characteristic granular scale in the $10 \times 10 \times 1$ calculation, there is a peak in the spectrum (at all depths) at approximately $k=3$. We conclude that this peak corresponds to the mesogranular scale. Note that this scale is comparable to the width of the domain in the $4 \times 4 \times 1$ calculation, so clearly a large domain is essential if the aim is to study mesogranular behaviour. It is also worth noting here that preliminary hydrodynamic calculations in a $16 \times 16 \times 1$ domain produce mesogranules of a similar spatial scale to those that are observed in the $10 \times 10 \times 1$ domain. This suggests that the $10 \times 10 \times 1$ domain is large enough to produce mesogranules that are not constrained by the horizontal scale of the computational domain. The results of Cattaneo et al. (2001) demonstrate that there is a similarly well-defined mesoscale in turbulent Boussinesq convection.

Further insight into the nature of the mesogranules can be gained by analysing the coherence times of the flow at different spatial scales. Given that there are no peaks in the spectra corresponding to a specific granular scale, it is not immediately obvious how to separate the granular motions from the mesogranular motions. A relatively crude way of doing this is to filter the velocity field in Fourier space, according to the horizontal wavenumber, using a high-pass $(k>4)$ and a low-pass $(1<k<4)$ filter. The threshold of $k=4$ is somewhat arbitrary, but is chosen so that the mesogranular scale is clearly contained in the low wavenumber range. Using each of the filtered flows, we calculate a mean (horizontally-averaged) correlation time for the horizontal components of the velocity field at $z=0.1$. This method gives a correlation time for the high-pass filtered velocity field of approximately 3.3 crossing times. Unsurprisingly, this correlation time for the small-scale motions is comparable to the convective turnover time, $\tau_{c}$. On the other hand, the correlation time for the low-wavenumber part of the velocity field is approximately 14.1 crossing times. 


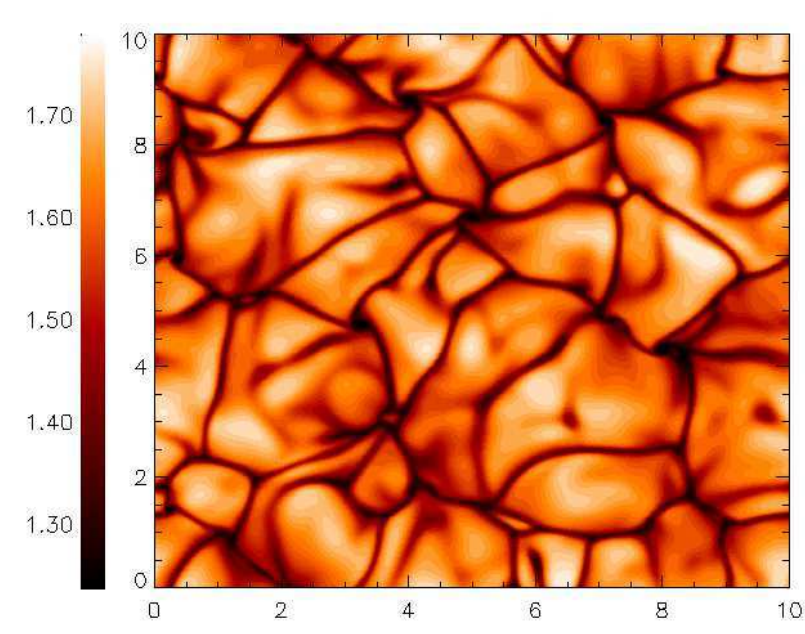

(a) Temperature at $z=0.1$

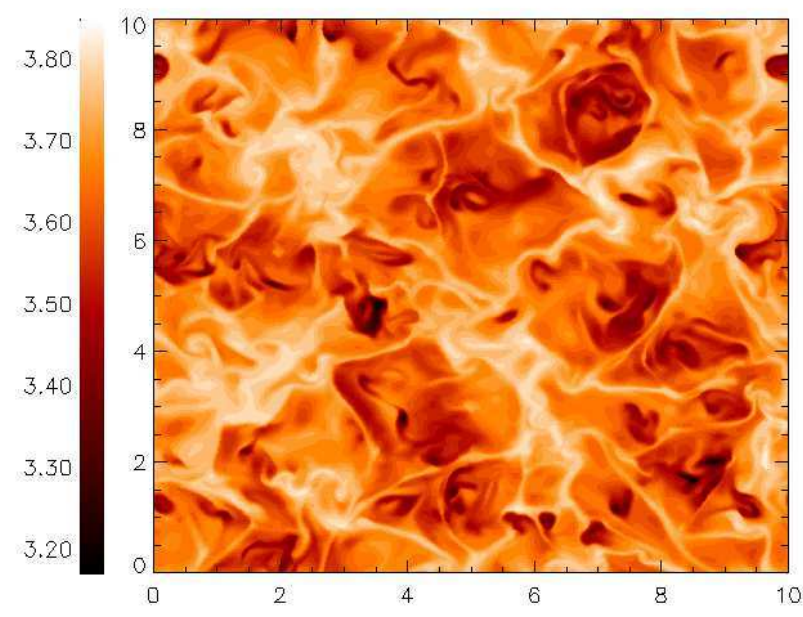

(c) Temperature at $z=0.9$

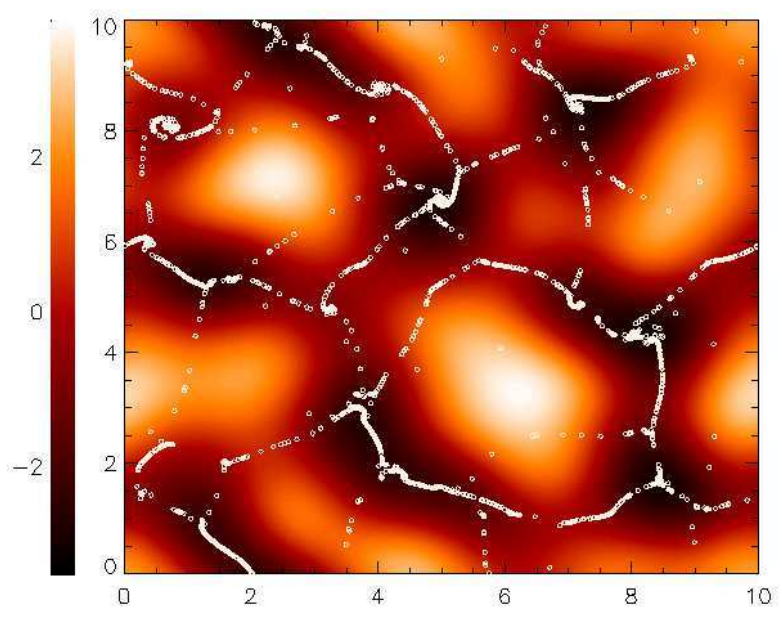

(b) Horizontal divergence and cork distribution at $z=0.1$

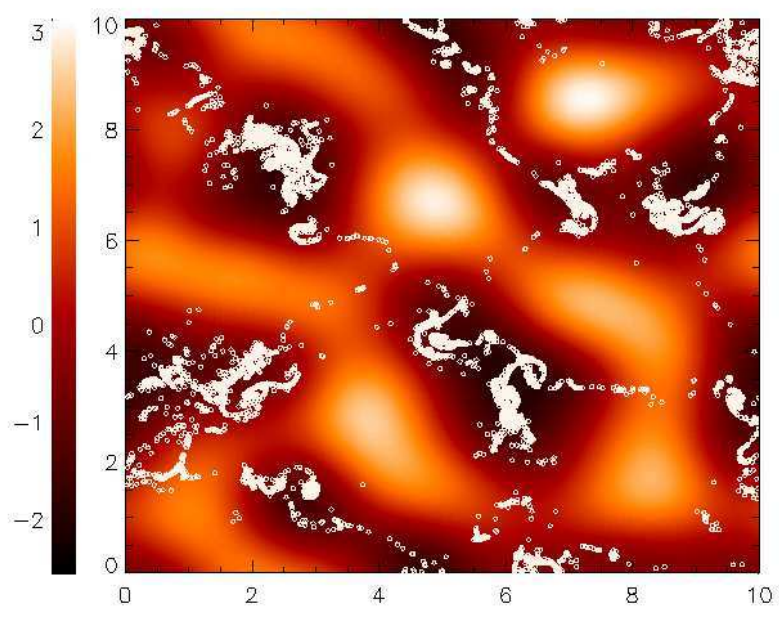

(d) Horizontal divergence and cork distribution at $z=0.9$

Figure 3. (colour online) Hydrodynamic convection with the addition of passive corks. This figure shows the state of the system at $t=239$, approximately two convective turnover times after the corks were introduced (at $t=232$ ). Left column: The temperature distribution in two different horizontal planes. Right column: The coarse-grained horizontal divergence in the same horizontal planes. Here bright (dark) regions correspond to regions of divergence (convergence). The white points indicate the location of each cork at this instant in time.

So, although we have made a rather arbitrary division between large and small scales to calculate the coherence times of the flow, this analysis indicates that the mesogranular motions evolve over a much longer time-scale than the smaller-scale granular flows.

The influence of the mesogranules can be illustrated by considering the evolution of passive tracer particles in the flow. Taking the convective state that is shown in Figure 1, we introduce particles (or "corks") into the flow at a particular depth. Following Cattaneo et al. (2001), we allow these particles to move horizontally, but not in the vertical direction. Hence, the trajectories of these particles are computed using the following equation:

$$
\frac{\partial \boldsymbol{x}_{p}}{\partial t}=\boldsymbol{u}_{H}\left(\boldsymbol{x}_{p}, t\right)
$$

where $\boldsymbol{x}_{p}$ is the position of the particle and $\boldsymbol{u}_{H}$ the horizontal velocity. The velocity at the particle position is interpolated from the grid values using a sixth-order Lagrangian interpolation scheme. The time-stepping 
scheme that is used to solve equation (7) is the same as for the other governing equations. Initially, the particles are distributed uniformly at two different depths: $z=0.1$ (near the upper boundary) and $z=0.9$ (near the lower boundary). We then follow the evolution of the particles and the flow for just over two convective turnover times. If, after this brief phase of evolution, the particles outline a mesogranular network, it is reasonable to assume that regions of vertical magnetic flux in a dynamo calculation should accumulate on similar scales.

The state of this system after evolving the equations for a couple of convective turnover times is illustrated in Figure 3, which shows the temperature distribution and the particle positions in the two horizontal planes corresponding to $z=0.1$ and $z=0.9$. Following Cattaneo et al. (2001), we also calculate the horizontal divergence, $\boldsymbol{\nabla} \cdot \boldsymbol{u}_{H}$, at each depth, convolving the result with a Gaussian filter to highlight the large-scale motions. In both layers, there is a large scale pattern of converging and diverging regions. This corresponds to the mesogranular motions within the domain. As expected, particles tend to accumulate in the converging regions on a mesogranular scale. Near the surface, the particle clustering outlines a welldefined network of convective downflows on a scale that is clearly larger than the basic granular scale. Near the lower boundary, where the flow is more turbulent, the particles again form a complementary pattern of persistent clusters, but these accumulations are rather dispersed compared to the coherent network that is observed in the upper layers. The results of Cattaneo et al. (2001) suggest that we should expect to see an enhanced level of clustering at later times, further highlighting the presence of mesogranules. It is also worth noting here that clustering on the mesogranular scale does not occur in the same way if one allows the particles to be advected by the total velocity rather than just the horizontal velocity. Of course, the mesogranular motions are still present in that case, but the particles are always able to escape from the two-dimensional regions of horizontal convergence by moving in a direction that is perpendicular to the plane.

\subsection{Dynamo properties}

In the previous section, it was established that the basic hydrodynamic state in this model produces nearsurface granular convection, with a clearly-defined mesogranular scale. So, although this model is rather idealised it does appear to be a reasonable representation of convection in the quiet Sun. In order to investigate the dynamo properties of this flow, we insert a seed vertical magnetic field into the convective state that is illustrated in Figure 1. In this $10 \times 10 \times 1$ computational domain, this initial field is given by:

$$
B_{z}=B_{0} \cos \left(\frac{2 \pi x}{10}\right) \cos \left(\frac{2 \pi y}{10}\right)
$$

where $B_{0}$ gives the peak amplitude of the field. In these non-dimensional units, a value of $B_{0}=0.007$ implies that this initial magnetic energy is approximately $0.004 \%$ of the total kinetic energy of the flow. As previously discussed, the evolution of this seed field depends crucially upon the value of $\zeta_{0}$ (or, equivalently, $\mathcal{R} m$ ), which can be set as the field is introduced.

Figure 4 shows the evolution of the total magnetic energy, as a function of time, for the three values of $\zeta_{0}$ that are specified in Table 1. In all three simulations, the magnetic energies have been normalised by the mean value of the kinetic energy during the final stages of the highest $\mathcal{R} m$ calculation (which is also shown, for reference). Regardless of the value of the magnetic Reynolds number, the magnetic energy grows during the early stages of the simulation. During this phase, this increase in the magnetic energy is due to the fact that vertical flux is expelled from the granular interiors, forming magnetic flux concentrations in the convective downflows. After this initial phase of transient growth, the magnetic Reynolds number starts to play an important role. In the lowest $\mathcal{R} m$ case, $\mathcal{R} m=115$, the magnetic energy decays, which implies that there is no dynamo action. Note that the decay of magnetic energy in this case is not a smooth exponential. This is partly due to the fact that the field is locally dynamically significant during the early stages of decay, but the shape of the decay curve is also strongly influenced by small-scale fluctuations in the flow. Of course, it would be possible (in theory) to determine a decay rate by continuing to evolve this 


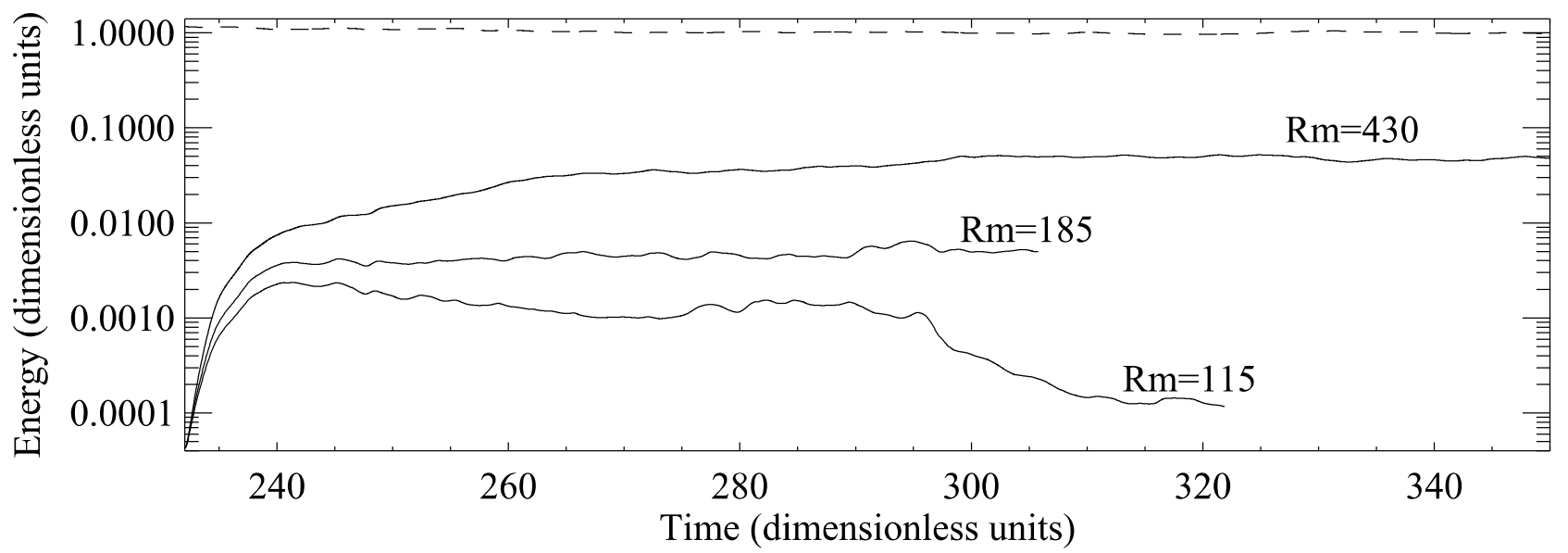

Figure 4. The evolution of the dynamo calculations for three different values of $\mathcal{R} m: \mathcal{R} m=430\left(\zeta_{0}=0.15\right), \mathcal{R} m=185\left(\zeta_{0}=0.35\right)$ and $\mathcal{R} m=115\left(\zeta_{0}=0.55\right)$. The dashed line shows the time-dependence of the kinetic energy in the highest $\mathcal{R} m$ case. The solid lines show the evolution of the magnetic energy for each case. All of these energy curves have been normalised by the time-average of the kinetic energy curve over the final 50 time units (corresponding to approximately 16 convective turnover times).

calculation. However, the successful dynamo calculations are of greater interest in this particular study, so this was not done here. For $\mathcal{R} m=185$, the magnetic energy increases very gradually with time after the initial phase of transient growth. We conclude from this that $\mathcal{R} m=185$ is just above the critical magnetic Reynolds number for the onset of dynamo action. In an equivalent calculation in a $4 \times 4 \times 1$ domain (Bushby et al. 2011), the critical magnetic Reynolds number was found to be approximately $\mathcal{R} m=325$. So it seems that dynamo action is easier to excite in a larger domain in this region of parameter space. Returning to Figure 4, it is clear that dynamo action is present in the highest $\mathcal{R} m$ case, $\mathcal{R} m=430$. Due to the fact that the magnetic field rapidly becomes dynamically significant, the kinematic regime (in which the magnetic field grows exponentially) is too brief in this particular calculation to allow an accurate determination of the kinematic growth rate. A separate calculation, in which the Lorentz force terms in the code were switched off, yielded a growth rate of 0.066 (in non-dimensional units). Comparing this with an equivalent calculation in a $4 \times 4 \times 1$ domain, which produced a growth rate of 0.026 (Bushby et al. 2011), we have further evidence to support the idea that (kinematic) dynamo action is more efficient in the larger domain. After the very short kinematic phase, the $\mathcal{R} m=430$ calculation enters an extended phase of nonlinear growth before finally saturating in the nonlinear regime. In the saturated state the magnetic energy is approximately $5 \%$ of the final kinetic energy. This saturation level is similar to that found in a comparable $4 \times 4 \times 1$ nonlinear dynamo calculation.

It is natural to conclude from these results that it is the mesogranular (rather than the granular) motions that are responsible for determining the kinematic growth rate of the dynamo in the $10 \times 10 \times 1$ domain. Clearly a $4 \times 4 \times 1$ domain is not large enough to accommodate mesogranules, which presumably explains the higher critical magnetic Reynolds number (and lower kinematic growth rates) in that case. As we have already discussed, there is no well-defined granular scale, so this is not a (comparatively) simple two-scale flow of the type considered in the kinematic dynamo study of Cattaneo \& Tobias (2005). However, given the presence of (relatively) coherent mesogranules, there are some parallels between this model and that considered by Tobias \& Cattaneo (2008), who investigated the effects that coherent structures have upon a turbulent dynamo. They found that the kinematic dynamo growth rates dropped considerably when the "active dynamo scales" were filtered out of the velocity field. Although it is not feasible to carry out a similar study for this complex convective flow, the observed dependence upon the domain size suggests that the active dynamo scales in the $10 \times 10 \times 1$ case are comparable to the mesogranular scale. In physical terms, this could be due to the fact that longer-lived magnetic flux concentrations are able to accumulate preferentially at the mesogranular boundaries in the larger domain. On the other hand, the rapidly evolving small-scale convective pattern in the $4 \times 4 \times 1$ domain continuously mixes regions of opposing magnetic polarities, which may explain why it is more difficult to excite a dynamo in that case. It is interesting to note that the beneficial influence of mesogranules seems to be solely a kinematic effect: there is no evidence 


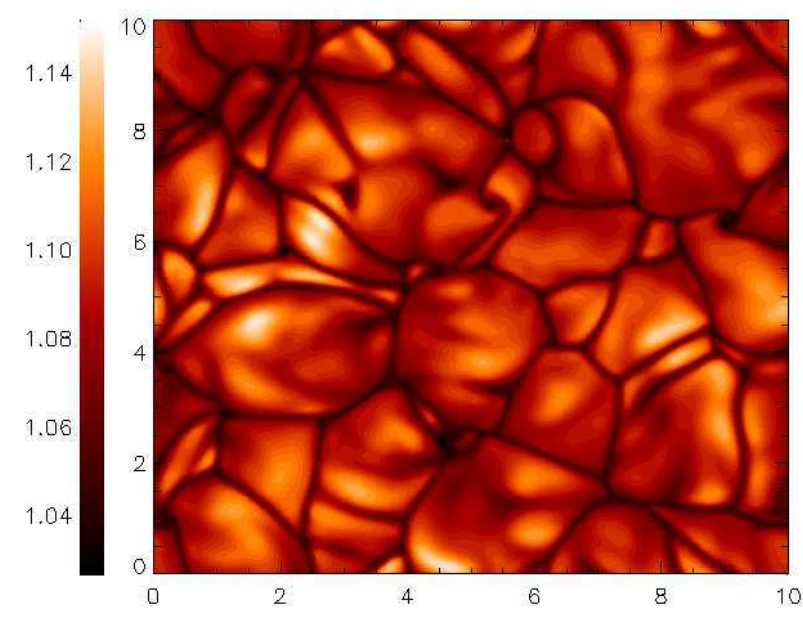

(a) Temperature at $z=0.02$

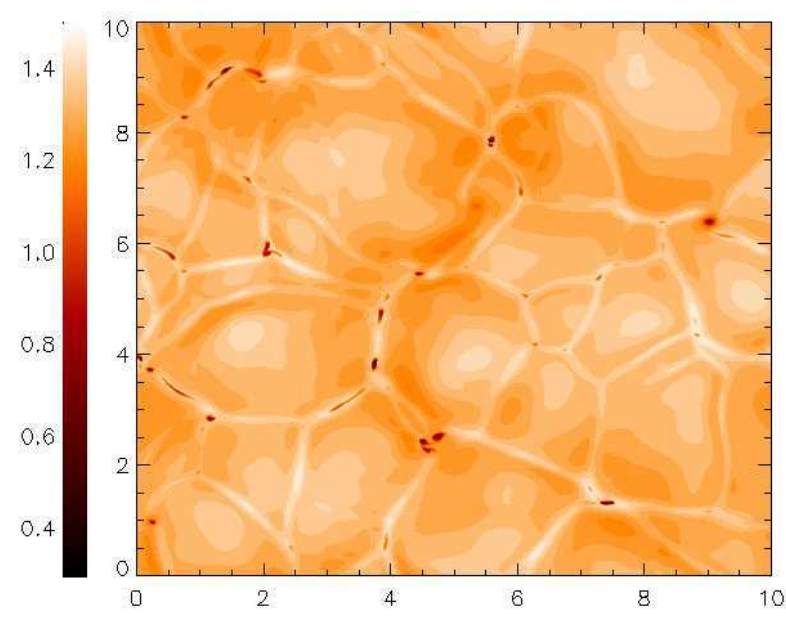

(c) Density at $z=0.02$

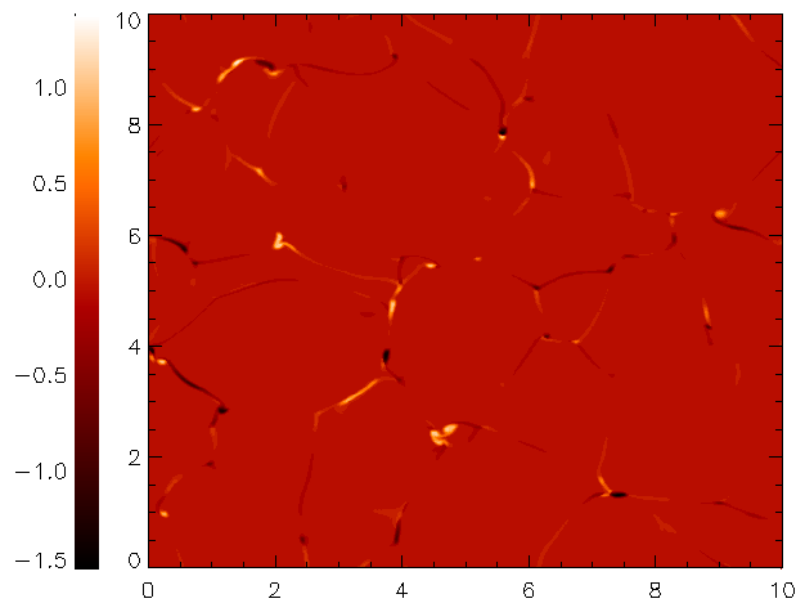

(b) Vertical magnetic field at $z=0.02$

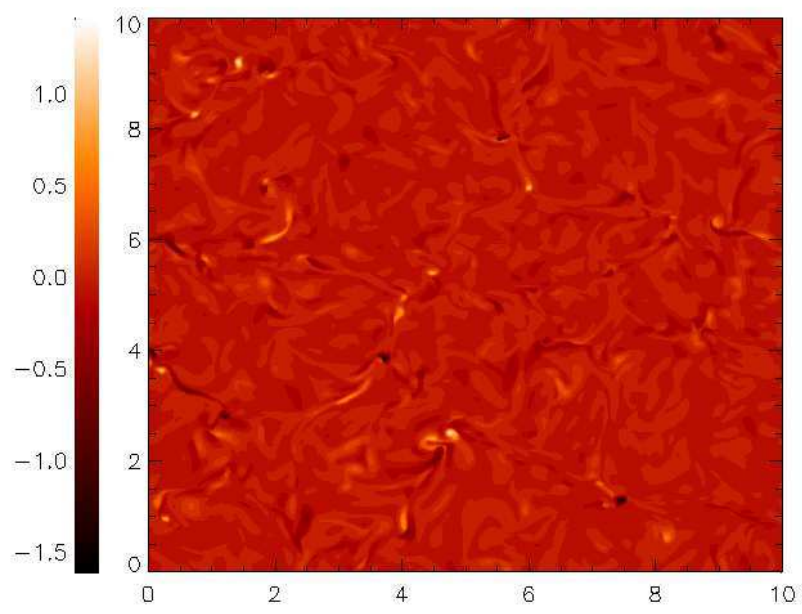

(d) Vertical magnetic field at $z=0.5$

Figure 5. (colour online) A snapshot of the saturated dynamo in the $\mathcal{R} m=430$ case at $t=339$ (approximately 107 time units after the magnetic field was first introduced). The plots on the left show the temperature and density distributions near the upper surface. The plots on the right show the horizontal distribution of $B_{z}$ at $z=0.02$ and $z=0.5$.

from these calculations to suggest that the presence of mesogranules alters the nonlinear efficiency of the dynamo.

Figure 5 shows the state of the $\mathcal{R} m=430$ calculation during the saturated nonlinear phase. In qualitative terms, the temperature distribution near the upper surface is virtually indistinguishable from the hydrodynamic state that is illustrated in Figure 1. However, magnetic fields are present, forming mixed polarity concentrations of vertical flux in the convective downflows near the surface of the domain. As expected, these magnetic flux concentrations do appear to accumulate preferentially on a mesogranular scale. Given that the temperature plot looks rather similar to hydrodynamic convection, we can conclude that the dynamical influence that these magnetic fields have upon the flow must be rather localised. This is illustrated clearly in the density plot, where regions of low density correspond to regions of high magnetic pressure. As discussed in the Introduction, the partial evacuation of magnetic regions in models of this type is well understood, and this phenomenon plays an important role in determining the peak magnetic field strengths in these vertical magnetic flux concentrations. Prompted by the rather 'point-like' nature of the partially-evacuated regions near the surface of the domain, it is natural to relate these to the bright points that are associated with strong magnetic flux concentrations in G-band images of the solar surface. This is clearly not an unreasonable connection to make given that these regions correspond to areas of 


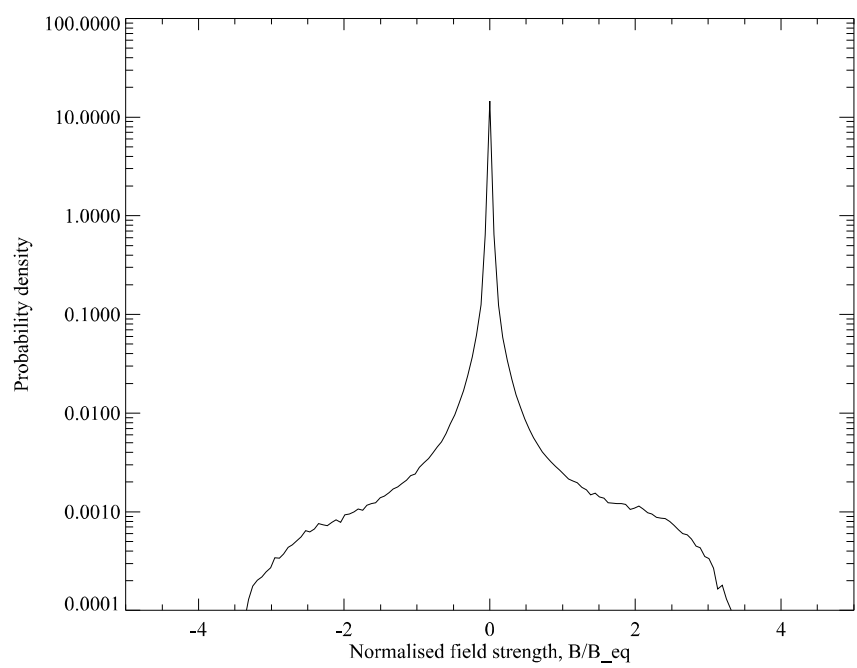

(a) $z=0.02$

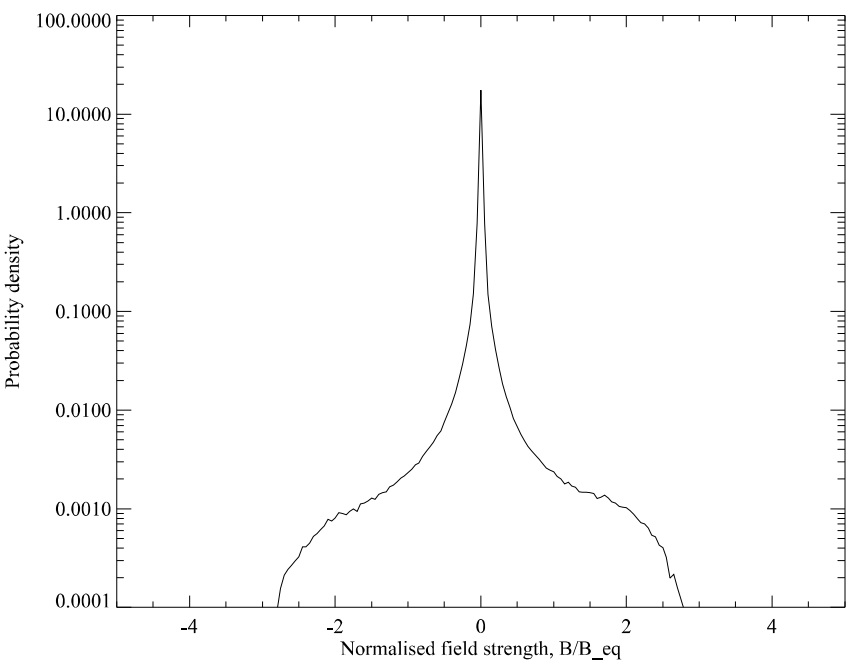

(b) $z=0.5$

Figure 6. Probability density functions for the vertical component of the magnetic field in two horizontal planes $(z=0.02$ and $z=0.5)$ for the $\mathcal{R} m=430$ case. These planes correspond to the magnetic field distributions that are shown in Figure 5 . In both cases, the magnetic field strength has been normalised by the equipartition field strength $B_{\text {eq }}$ (in dimensionless units $B_{e q}=0.415$ at $z=0.02$ and $B_{e q}=0.5$ at $z=0.5$ ). Each plot has been obtained by time-averaging the probability density functions over 35 time units (or just over 11 convective turnover times) during the nonlinear saturated phase.

high magnetic pressure in these simulations. At the mid-layer of the domain, the horizontal distribution of the strongest vertical magnetic flux concentrations again suggests a mesogranular scale.

The presence of partially evacuated magnetic regions near the surface of the domain suggests a high value for the local magnetic field strength. As discussed in the Introduction, magnetic field strengths can often exced a kilogauss in the quiet Sun. It is not really meaningful to discuss kilogauss-strength magnetic fields in the context of this idealised, dimensionless model. However, we can relate the peak magnetic energy density to the mean kinetic energy density of the surrounding convective motions. At each depth, we can define the equipartition field strength as follows:

$$
B_{e q}=\sqrt{\left\langle\rho|\boldsymbol{u}|^{2}\right\rangle}
$$

where $\langle$.$\rangle denotes a horizontal average. In each layer, field strengths in excess of this equipartition value$ correspond to magnetic energy densities that are larger than the horizontally-averaged kinetic energy density of the convection. Figure 6 shows probability density functions for the vertical component of the magnetic field (normalised by $B_{e q}$ ) at $z=0.02$ and $z=0.5$, for the $\mathcal{R} m=430$ case. Both probability density functions take the form of a stretched exponential distribution (except at high field strengths where there is a clear "cut off" in the probability density functions), with most of the domain being field free. This is consistent with previous studies of dynamo action in compressible convection (see, for example, Vögler \& Schüssler 2007). The peak fields near the surface are (approximately) 3 times larger than the equipartition value. Given that $B_{e q}$ at the solar surface is estimated to be approximately 400G (Galloway et al. 1977), these super-equipartition field strengths would correspond to the observed kilogauss-strength magnetic fields in the quiet Sun. Furthermore, larger values of $\mathcal{R} m$ should produce stronger fields (see, for example, Bushby et al. 2008). It is interesting to note that the probability density function for $z=0.5$ is rather similar to the near-surface plot. Hence, super-equipartition magnetic fields are not solely a near-surface phenomenon in dynamo models of this type. 


\section{Conclusions}

We have demonstrated the existence of both granular and mesogranular motions in an idealised model of convection near the solar surface. In a large aspect ratio domain, this convective flow acts as a small-scale dynamo provided that the magnetic Reynolds number exceeds a critical value of approximately $\mathcal{R} m=185$. This critical value is much smaller than that found in an otherwise identical dynamo calculation that was carried out in a narrower domain. We conclude from this that dynamo action is easier to excite in the larger domain, where mesogranules are present. Near the upper surface of the computational domain, the mesoscale pattern of mixed polarity magnetic flux concentrations compares favourably to solar observations (as well as the Boussinesq study of Cattaneo et al. 2001). Furthermore, the peak field strengths in these regions significantly exceed the equipartition value, something that is consistent with the measurement of kilogauss-strength magnetic fields in the quiet Sun. Where strong fields occur, the upper layers of the magnetic feature are always partially evacuated (indeed, a reduction in the internal gas pressure is the only way to satisfy local pressure balance in regions of high magnetic pressure). So, although Boussinesq calculations do appear to produce a similar magnetic field distribution, strongly super-equipartition fields can only be generated if the effects of compressibility are included in the dynamo model.

These results tend to support the hypothesis that a significant fraction of the quiet Sun magnetic fields is generated locally by the near-surface convective motions, particularly given that the magnetic Reynolds number at the solar photosphere is very large (an estimate of $\mathcal{R} m \approx 10^{6}$ is given by Ossendrijver 2003). However, it should be stressed that it is difficult to make definitive statements on this subject purely on the basis of idealised numerical experiments. Due to numerical constraints, the Reynolds numbers that can be obtained, even in local simulations, are rather modest. Estimates suggest that $\mathcal{R} e \approx 10^{12}$ in the solar photosphere (Ossendrijver 2003), which is nearly 10 orders of magnitude larger than the value of $\mathcal{R} e$ found in these simulations. One important consequence of this is that the magnetic Prandtl number, $P m=\mathcal{R} m / \mathcal{R} e$, which is of order unity in these dynamo calculations, is very small in the solar photosphere. Although this issue has not been explored in any detail in convectively-driven dynamos, turbulent dynamos tend to be rather inefficient in the low Pm regime (see, for example, Boldyrev \& Cattanco 2004, Schekochihin et al. 2005). This is certainly an issue that merits further investigation in the context of compressible convection. Another criticism that can be made of idealised models of this type is that the boundary conditions are rather artificial. This is particularly true of the impermeable lower boundary adopted here. Stein et al. (2003) attributed a lack of dynamo action in their local Cartesian model to the fact that their open lower boundary condition allowed convective downdrafts to advect magnetic flux out of their convective domain. However, this conclusion would appear to be inconsistent with the results of Brummell et al. (2010), who studied dynamo action in a two layer model, with a stable layer underneath a convectively unstable region. They found that the dynamo efficiency in the upper layer was in no way impaired by the presence of the stable layer. So, the fact that the lower boundary is closed in the present set of simulations probably does not have a major bearing upon the dynamo efficiency in the convective layer.

Acknowledgements PJB and BF would like to thank EPSRC for financial support (grant reference $\mathrm{EP} / \mathrm{H} 006842 / 1)$. We would also like the thank the two anonymous referees for their comments and suggestions. The numerical calculations that are described in this paper were carried out using the UKMHD consortium cluster and the HECToR supercomputing facility.

\section{REFERENCES}

Abbett, W.P., The magnetic connection between the convection zone and corona in the quiet Sun. Astrophys. J. 2007, 665, 1469-1488.

Boldyrev, S. and Cattaneo, F., Magnetic-field generation in Kolmogorov turbulence. Phys. Rev. Lett. 2004, 92, 144501.

Brummell, N.H., Tobias, S.M. and Cattaneo, F., Dynamo efficiency in compressible convective dynamos with and without penetration. Geophys. Astrophys. Fluid Dyn. 2010, 104, 565-576. 
Bushby, P.J., Houghton, S.M., Proctor, M.R.E. and Weiss, N.O., Convective intensification of magnetic fields in the quiet Sun. Mon. Not. Roy. Astron. Soc. 2008, 387, 698-706.

Bushby, P.J., Proctor, M.R.E. and Weiss, N.O., Small-scale dynamo action in compressible convection. Astron. Soc. Pac. Conf. Series 2010, 429, 181-186.

Bushby, P.J., Proctor, M.R.E. and Weiss, N.O., The influence of stratification upon small-scale convectively-driven dynamos. In Astrophysical dynamics: from stars to galaxies, Proceedings of the IAU Symposium No. 271, edited by N.H. Brummell, A.S. Brun, M.S. Miesch and Y. Ponty, 2011, in press.

Cattaneo, F., On the origin of magnetic fields in the quiet photosphere. Astrophys. J. 1999, 515, L39-L42.

Cattaneo, F., Lenz, D. and Weiss, N.O., On the origin of the solar mesogranulation. Astrophys. J. 2001, 563, L91-L94.

Cattaneo, F. and Tobias, S.M., Interaction between dynamos at different scales. Phys. Fluids 2005, 17, 127105 .

de Wijn, A.G., Stenflo, J.O., Solanki, S.K. and Tsuneta, S., Small-scale solar magnetic fields. Space Sci. Rev. 2009, 144, 275-315.

Domínguez Cerdeña, I., Evidence of mesogranulation from magnetograms of the Sun. Astron. Astrophys. 2003, 412, L65-L68.

Domínguez Cerdeña, I., Sánchez Almeida, J. and Kneer, F., Quiet Sun magnetic fields from simultaneous inversions of visible and infrared spectropolarimetric observations. Astrophys. J. 2006, 646, 1421-1435.

Galloway, D.J., Proctor, M.R.E. and Weiss, N.O., Formation of intense magnetic fields near the surface of the Sun. Nature 1977, 266, 686-689.

Käpylä, P.J., Korpi, M.J. and Brandenburg, A., Large-scale dynamos in turbulent convection with shear. Astron. Astrophys. 2008, 491, 353-362.

Khomenko, E.V., Shelyag, S., Solanki, S.K. and Vögler, A., Stokes diagnostics of simulations of magnetoconvection of mixed-polarity quiet-Sun regions. Astron. Astrophys. 2005, 442, 1059-1078.

Lin, H. and Rimmele, T., The granular magnetic fields of the quict Sun. Astrophys. J. 1999, 514, 448-455.

Matthews, P.C., Proctor, M.R.E. and Weiss, N.O., Compressible magnetoconvection in three dimensions: planforms and nonlinear bchaviour. J. Fluid Mech. 1995, 305, 281-305.

Meneguzzi M. and Pouquet A., Turbulent dynamos driven by convection. J. Fluid Mech. 1989, 205, 297318.

Muller, R., Auffret, H., Roudier, T., Vigneau, J., Simon, G.W., Frank, Z., Shine, R.A. and Title, A.M., Evolution and advection of solar mesogranulation. Nature 1992, 356, 322-325.

Nagata, S., Tsuncta, S., Sucmatsu, Y., Ichimoto, K., Katsukawa, Y., Shimizu, T., Yokoyama, T., Tarbell, T.D., Lites, B.W., Shine, R.A., Berger, T.E., Title, A.M., Bellot Rubio, L.R. and Orozco Suárez, D., Formation of solar magnetic flux tubes with kilogauss field strength induced by convective instability. Astrophys. J. Lett. 2008, 677, L145-L147.

November, L.J., Toomre, J., Gebbic, K.B. and Simon, G.W., The detection of mesogranulation on the sun. Astrophys. J. 1981, 245, L123-L126.

Ossendrijver, M., The solar dynamo. Astron. Astrophys. Rev. 2003, 11, 287-367.

Parnell, C.E., DeForest, C.E., Hagenaar, H.J., Johnston, B.A., Lamb, D.A. and Welsch, B.T., A power-law distribution of solar magnetic fields over more than five decades in flux. Astrophys. J. 2009, 698, 75-82.

Pietarila Graham, J., Cameron, R. and Schüssler, M., Turbulent small-scale dynamo action in solar surface simulations. Astrophys. J. 2010, 714, 1606-1616.

Rieutord, M. and Rincon, F., The Sun's supergranulation. Living Rev. Solar Phys. 2010, 7, 2 (http://www.livingreviews.org/lrsp-2010-2).

Rincon, F., Lignières, F. and Rieutord, M., Mesoscale flows in large aspect ratio simulations of turbulent compressible convection. Astron. Astrophys. 2005, 430, L57-L60.

Sánchez Almeida, J., Bonet, J.A., Viticchié, B. and Del Moro, D., Magnetic bright points in the quiet Sun. Astrophys. J. Lett. 2010, 715, L26-L29.

Schekochihin, A.A., Haugen, N.E.L., Brandenburg, A., Cowley, S.C., Maron, J.L. and McWilliams, J.C., The onset of a small-scale turbulent dynamo at low magnetic Prandtl numbers. Astrophys. J. 2005, 625, L115-L118.

Schüssler, M. and Vögler, A., Strong horizontal photospheric magnetic field in a surface dynamo simulation. 
Astron. Astrophys. 2008, 481, L5-L8.

Shine, R.A., Simon, G.W. and Hurlburt, N.E., Supergranule and mesogranule evolution. Solar Physics 2000, 193, 313-331.

Spruit, H.C., Convective collapse of flux tubes. Solar Phys. 1979, 61, 363-378.

Stein, R.F., Bercik, D. and Nordlund, A., Solar surface magneto-convection. Astron. Soc. Pac. Conf. Series 2003, 286, 121-131.

Stein, R.F. and Nordlund, A., Solar small-scale magnetoconvection. Astrophys. J. 2006, 642, 1246-1255.

Stix, M., The Sun: An Introduction (Springer, Berlin), 2004.

Tobias, S.M. and Cattaneo, F., Dynamo action in complex flows: the quick and the fast. J. Fluid Mech. 2008, 601, 101-122.

Tobias, S.M., Cattaneo, F. and Brummell, N.H., Convective dynamos with penetration, rotation and shear. Astrophys. J. 2008, 685, 596-605.

Vögler, A. and Schüssler, M., A solar surface dynamo. Astron. Astrophys. 2007, 465, L43-L46.

Weiss, N.O., The expulsion of magnetic flux by eddies. Proc. Roy. Soc. London A 1966, 293, 310-328.

Yelles Chaouche, L., Moreno-Insertis, F., Martínez Pillet, V., Wiegelmann, T., Bonet, J.A., Knölker, M., Bellot Rubio, L.R., Del Toro Iniesta, J.C., Barthol, P., Gandorfer, A., Schmidt, W. and Solanki, S.K., Mesogranulation and the solar surface magnetic field distribution, Astrophys. J. Lett. 2011, 727, L30. 